\title{
Perfiles de Velocidad en el Cilindro de un Motor Alternativo
}

\author{
Simón J. Fygueroa, Jesús O. Araque y Carlos G. Villamar \\ GRUMOTE. Universidad de Los Andes, Facultad de Ingeniería, Núcleo Pedro Rincón Gutiérrez, \\ La Hechicera, Edif. B. Of. 2E10, Mérida, Mérida-Venezuela (e-mail: figueroa@ula.ve)
}

\section{Resumen}

El objetivo del presente trabajo es mostrar los resultados de la medición de las componentes de la velocidad del aire admitido en el cilindro simulado de un motor CFR (Cooperative Fuels Research) instalado en un banco de flujo estacionario al cual se ha conectado un sistema de adquisición de datos. Para determinar las velocidades se empleó un anemómetro de hilo caliente previamente calibrado. Se realizaron mediciones sobre diferentes puntos de varios planos transversales del cilindro para diferentes levantamientos de la válvula de admisión. Como resultado de las pruebas se obtuvieron los perfiles de las velocidades sobre los planos considerados. A partir de estos perfiles se evidencia la presencia de vórtices o torbellinos de evolución axial y de evolución radial en el interior del cilindro.

Palabras clave: motor CFR, banco de flujo, anemómetro, vórtice, torbellino

\section{Velocity Profiles in an Alternative Engine Cilinder}

\begin{abstract}
The present work objective is to show experimental results for speed components of air flow admitted in a CFR engine simulated cylinder (Cooperative Fuels Research), installed in a stationary flow bench provided with a data acquisition system. The speed components were measured using a calibrated hot wire anemometer. Measurements were made for different admission valve lifts on different points on several cylinder cross-sectional planes. As a result, the speed profiles on the planes considered in the study were obtained. From these profiles the presence of swirl and tumble in the interior of the engine cylinder was verified.
\end{abstract}

Keywords: CFR engine, flow bench, anemometer, swirl, tumble 


\section{INTRODUCCIÓN}

Debido a que el desarrollo de los motores de combustión interna alternativos (MCIA) está condicionado por normas anticontaminación cada día más severas, y puesto que la reducción de las emisiones del motor de encendido por chispa $(\mathrm{MECH})$ se puede conseguir mediante un control mejorado de la homogeneidad de la mezcla aire-combustible, de la dispersión cíclica y de la intensidad de la turbulencia en el momento del encendido (Maier et al., 2000), fenómenos sobre los cuales influye grandemente el flujo dentro del cilindro, es muy importante una apropiada comprensión de como se inician, establecen, evolucionan y se destruyen los flujos dentro del cilindro. Por otra parte y como una aplicación más reciente, uno de los métodos para incrementar la eficiencia exergética de un $\mathrm{MECH}$ es lograr una adecuada mejora del proceso de mezclado del aire con el combustible (Rakopoulos y Giakoumis, 2006).

Los primeros estudios referentes al movimiento del aire en MCIA fueron realizados por Tanaka (1929), quien estableció los cuatro regímenes de flujo que se presentan alrededor de la válvula de admisión. Desde esta época se han realizado innumerables publicaciones sobre el tema; una amplia y conocida revisión al respecto hasta la fecha de su edición, se encuentra en la obra póstuma de Benson (1986)

A causa de que las condiciones de trabajo en el interior del cilindro del MCIA son tan hostiles que no permiten mediciones en funcionamiento, desde los inicios los investigadores han empleado para sus ensayos bancos de flujo estacionario (Hong y Tarng, 2001; Yun, 2002) en los cuales se parte de la suposición que el campo de velocidades no varía con el tiempo pero si espacialmente.

Existen dos enfoques para analizar y predecir el flujo en el interior del cilindro. Un enfoque global basado en modelos simples (cero y cuasidimensionales) que dan información limitada pero útil, sobre los procesos implicados como por ejemplo, los modelos unidimensionales de compresión-expansión, el simétrico del movimiento radial hacia dentro (squish) de cámaras labradas en el pistón o el de cálculo del vórtice centrado en el eje del cilindro. El enfoque multidimensional permite determinar el campo de velocidades en las direcciones radial, axial y tangencial cuando se trata de estudios tridimensionales o de las dos primeras en los bidimensionales (Stickland et al., 2001).

Hay dos movimientos del aire que influyen grandemente sobre el campo de velocidades dentro del cilindro: los vórtices de evolución axial (swirl) y los vórtices de evolución radial (tumble) que han sido objeto de estudio por diferentes investigadores ( $\mathrm{Li}$ et al., 2002, Chang, 2004; Huang, 2005), que se evalúan de manera global mediante los coeficientes de vorticidad axial y tangencial. Actualmente se han definido nuevos índices que caracterizan mejor los movimientos vorticosos: Coeficiente de flujo angular, ángulo de inclinación, parámetro de flujo angular y ángulo de flujo (Yun, 2002)

Para identificar cuantitativamente el flujo en el cilindro en dos y tres dimensiones, inicialmente se uso anemometría de hilo caliente (HWA). Mas recientemente se usa anemometría láser Dopler (LDA) (Kang y Reitz, 2000; Hong y Tarng, 2001), velocimetría del rastro de partículas (PTV) (Lee et al., 2001) y velocimetría de la imagen de partículas (PIV) (Lee y Lee, 2003; Lee et al., 2005; Yasar, 2006).

El objetivo del presente trabajo es determinar el campo de velocidades que se produce en el interior del cilindro simulado de un motor CFR mediante anemometría de hilo caliente. Se emplea esta técnica porque el anemómetro además de poseer un volumen de medición pequeño tiene una respuesta de alta frecuencia que implica buena resolución espacial y temporal (Hutchins y Choi, 2002).

\section{FUNDAMENTOS DE LA ANEMOMETRÍA DE HILO CALIENTE}

Los anemómetros de hilo caliente son dispositivos para medir la velocidad de un gas. Para ello se usa una sonda en la cual va instalado un hilo metálico que se calienta por efecto Joule y se enfría por el flujo incidente, en función del gasto circulante y la temperatura del flujo, predominantemente mediante convección forzada. A partir de la diferencia de tensión entre los extremos del hilo o de su resistencia eléctrica (en el caso del anemómetro de intensidad constante) es posible entonces, obtener información de la velocidad del flujo. 


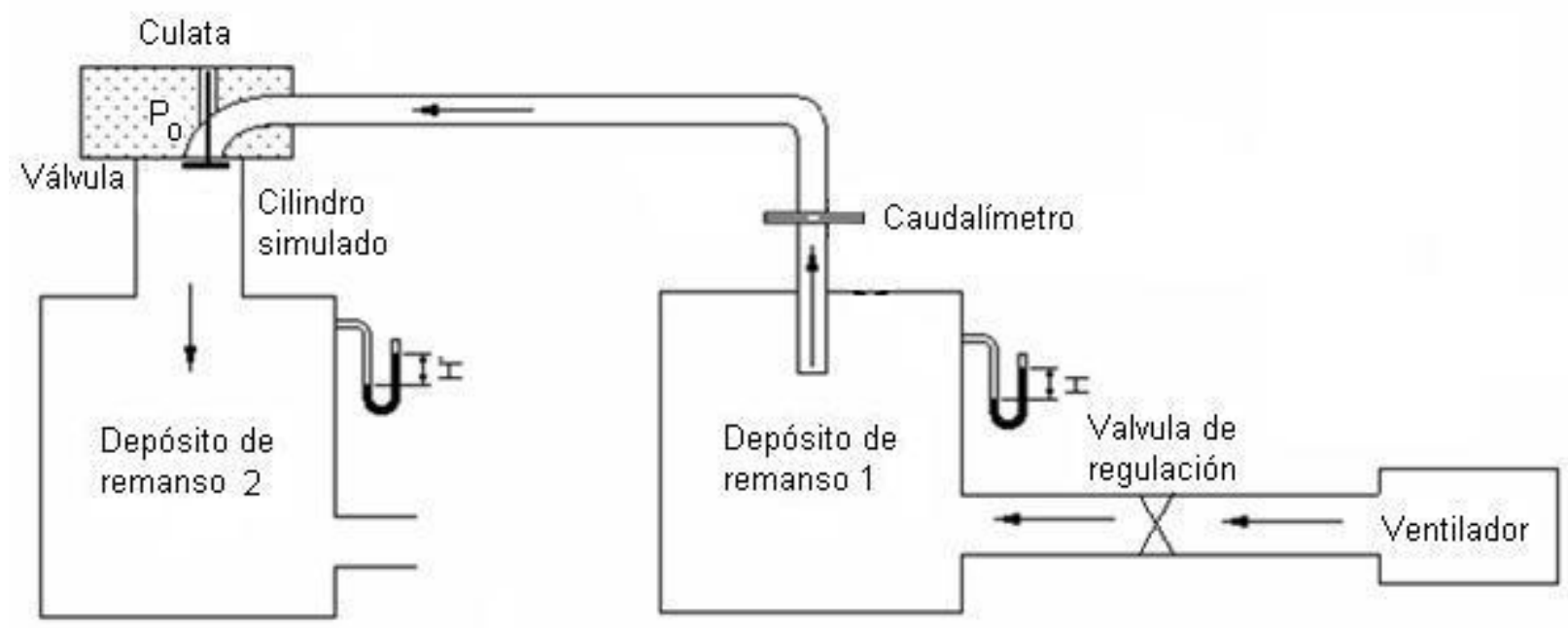

Fig. 1: Banco de flujo estacionario.

Los sensores de un anemómetro de hilo caliente son hilos con un diámetro de $0.5-5 \mu \mathrm{m}$ y una longitud de $0.1-2 \mathrm{~mm}$. Son por lo tanto, de un orden de magnitud análogo o incluso inferior al de los remolinos turbulentos más pequeños, con lo que se consigue mantener uniforme la distribución de la velocidad instantánea en la región ocupada por el sensor, al mismo tiempo que se minimizan enormemente las distorsiones ocasionadas por las sondas en los flujos. Los hilos se fabrican con materiales como tungsteno, platino o aleaciones de éste con otros materiales conductores como el iridio o el rodio. Los de tungsteno aunque son resistentes y tienen un elevado coeficiente de variación de la resistencia con la temperatura, no pueden utilizarse a altas temperaturas debido a su débil resistencia a la oxidación. Los de platino poseen una buena resistencia a la oxidación, un buen coeficiente de temperatura y son de fácil fabricación, pero son muy quebradizos a altas temperaturas. Las aleaciones de platino- iridio se sitúan en una posición intermedia entre el tungsteno y el platino.

\section{INSTALACIÓN}

La instalación utilizada consta de: banco de flujo estacionario, cilindro simulado, anemómetro de hilo caliente y tarjeta de adquisición de datos.

El banco de flujo estacionario empleado se muestra en la Fig. 1; el dispositivo generador de flujo es un ventilador centrífugo que impulsa el aire a través de un primer depósito de remanso que cumple la función de eliminar las fluctuaciones del flujo.
Un caudalímetro de membrana caliente (medidor MAF de un vehículo comercial) que produce una señal eléctrica que es captada y procesada por la tarjeta de adquisición de datos, permite determinar a partir de la curva de calibración del MAF, el gasto que circula por el sistema en tiempo real.

El aire que sale del caudalímetro es conducido por un tubo hacia la culata del motor CFR, luego pasa a través de su válvula de admisión a un cilindro simulado de metacrilato y descarga en el depósito de remanso final que atenúa las fluctuaciones de presión producidas por la apertura de la válvula.

Aguas arriba del primer depósito de remanso se encuentra una válvula de regulación de accionamiento manual que permite variar el caudal de aire impulsado, para fijar el salto de presiones a través de la válvula de admisión. El salto se determina a partir de las presiones

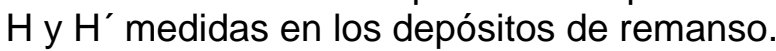

Para simular el levantamiento de la válvula de admisión se monta solidario a la culata del motor, un tornillo micrométrico que tiene un recorrido de $10 \mathrm{~mm}$ y permite obtener levantamientos de ésta en múltiplos de $0.125 \mathrm{~mm}$. El cilindro simulado de metacrilato transparente muy resistente, permite soportar el peso de la culata y además observar a través de él, el comportamiento del aire, de la sonda y de cualquier otro instrumento que se instale en su interior. Como la manipulación de la sonda (hilo caliente) es extremadamente delicada se seccionó el cilindro en varios planos para realizar las medidas, como se muestra en la Fig. 2. 

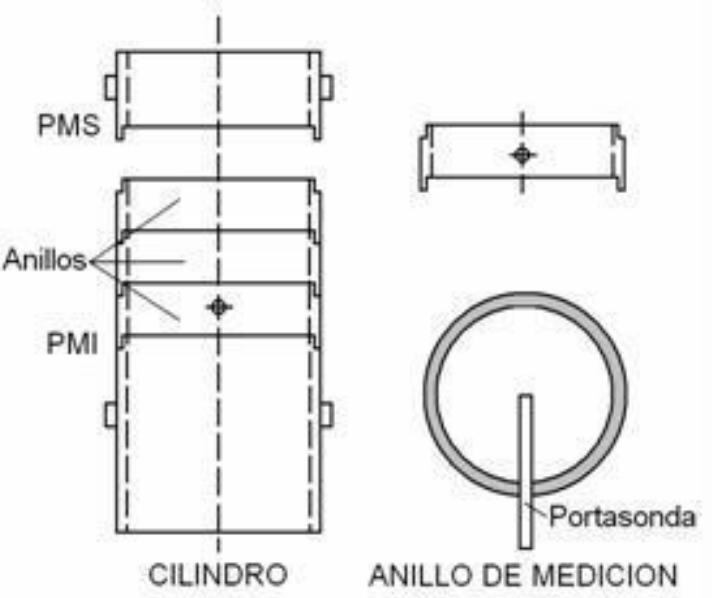

ANILLO DE MEDICION

Fig. 2: Cilindro simulado

Los anillos así obtenidos se pueden desplazar hacia arriba, hacia abajo y rotar. Sobre uno de estos anillos se instaló la sonda de manera definitiva para que estuviese fija de tal forma que para medir en diferentes planos solo se cambia de posición el anillo de medición sin tocar el sensor. Las dimensiones del cilindro son las siguientes: diámetro $82.55 \mathrm{~mm}$ (3.25"), espesor $5 \mathrm{~mm}$ y longitud $217 \mathrm{~mm}$.

El anemómetro de hilo caliente utilizado para la medición de las velocidades tiene las siguientes características: anemómetro de hilo caliente de temperatura constante marca TSI (Thermo Systems Inc.), modelo 1054-B conectado a un módulo de suministro de potencia TSI modelo 1051, para sensores simples (de un solo hilo) con dos salidas de tensión una libre o real (modo BRIDGE o en puente) y otra linealizada (modo LINEARIZED OUTPUT o de salida linealizada) que facilita la conversión del voltaje de salida a su equivalente en velocidad.

Para el anemómetro se obtuvo la curva de calibración que se muestra en la Fig. 3 y que

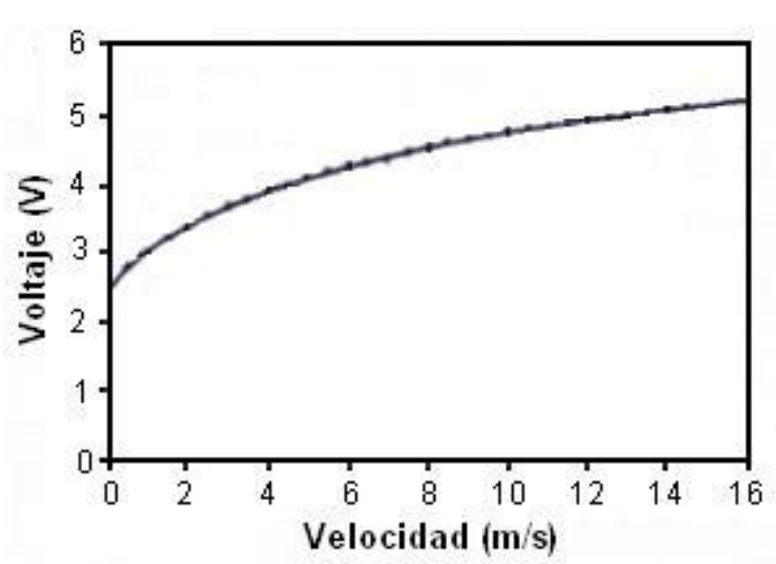

Fig. 3: Curva de calibración del anemómetro de hilo caliente

se puede representar mediante la ecuación:

$$
V=\frac{18.03+7.70 \mathrm{~V}^{0.77}}{7.49+\mathrm{V}^{0.77}}
$$

Donde $\mathcal{V}$ es la velocidad [m/s] y $\vee$ el voltaje de salida del anemómetro.

La adquisición de los datos de los ensayos se realiza con una tarjeta de adquisición de datos gestionada con el programa LabVIEW. La tarjeta que se instala al computador de la misma forma como se hace con cualquier periférico, posee la siguientes características: Fabricante: National Instrument Corporation, nombre: AT-MIO-ET, serie: ATE, tipo: DAQSIC y número de pines: 68

El instrumento virtual usado para la adquisición de datos es un multímetro digital de elevada precisión que calcula el valor promedio del voltaje obtenido del anemómetro a partir de 600 mediciones en un período máximo de 30 segundos. El programa de manejo además de este proceso, transforma la señal

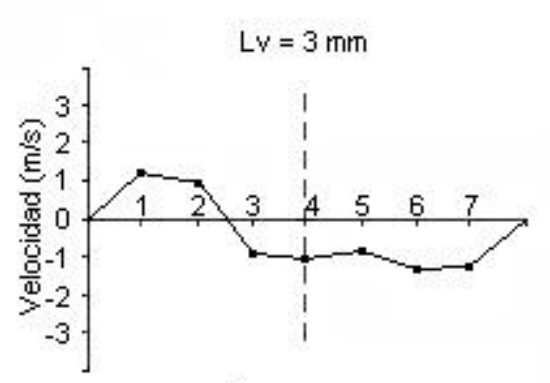

Posición de la sonda (cm)

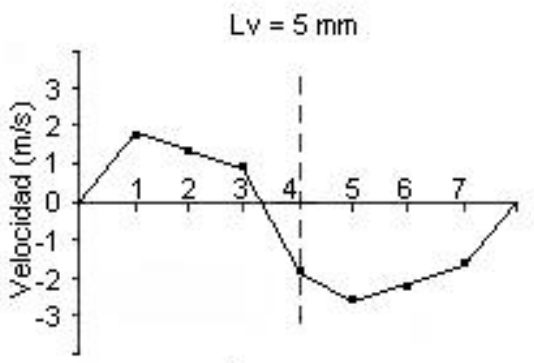

Posición de la sonda (cm)

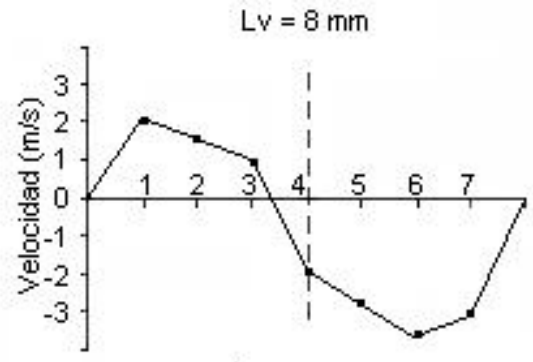

Posición de la sonda (cm)

tendencia

- - - eje del cilindro

Plano C, $P_{0}=10 \mathrm{~cm}$. c.a., Secc. 1-1'

Fig. 4: Distribución de velocidades tangenciales 
a su correspondiente valor de velocidad y almacena los resultados en archivos independientes, en el disco duro del computador para su posterior análisis.

\section{PROCEDIMIENTO}

En todos los ensayos llevados a cabo la temperatura se mantiene fija en $24^{\circ} \mathrm{C}$ y los valores de la presión absoluta Po antes de la válvula que se conservan fijos para las diferentes pruebas, son: $7 ; 10 ; 12.5$ y $15 \mathrm{~cm}$ de c.a. Para cada presión Po se efectúan medidas para levantamientos de la válvula con incrementos de $1 \mathrm{~mm}$. hasta el valor de máxima apertura $\mathrm{LV}=8 \mathrm{~mm}$. En resumen se realizan tres medidas de velocidad (axial, radial y tangencial) en catorce puntos de cuatro secciones para cuatro diferentes presiones y ocho levantamientos de válvula.

\section{RESULTADOS Y DISCUSIÓN}

Los resultados obtenidos presentan diferentes patrones de velocidad de los cuales se han seleccionado los de mayor frecuencia que por lo tanto son caracteristicos del movimiento del flujo de aire dentro del cilindro del motror CFR y se han representado en diagramas de velocidad que muestran la distribución espacial de las componentes axial y tangencial de la velocidad del aire.

En la distribución de velocidades tangenciales (Fig. 4) se observa que existe un remolino (vórtice de evolución axial) que se va desplazando hacia el centro a medida que se abre la válvula de admisión. Para bajos levantamientos de la válvula el flujo de aire se mueve irregularmente girando alrededor de un punto desplazado del eje del cilindro; pero a medida que se aumenta el área descubierta por la válvula de admisión se va desplazando el centro de rotación del flujo hasta situarse muy cerca del eje del cilindro. Esto se debe a la mayor capacidad de la geometría (área) del conducto para dirigir el flujo y provocar en el cilindro la mayor vorticidad posible.

De la distribución de velocidades axiales (Fig. 5) se deduce que para bajos levantamientos las velocidades debajo de la válvula de admisión son mayores que cerca de las paredes del cilindro, es decir en el sector más alejado del centro del remolino formado por el movimiento del flujo de aire. Este comportamiento se atribuye a que la descarga a través de la válvula se realiza mayormente en dirección radial por lo que el aire al chocar con las paredes del asiento de la válvula crea un flujo que trata de retornar al conducto de admisión. Esta característica se mantiene aún cuando la sección de medida se encuentre alejada de la culata. A medida que aumenta el levantamiento de la válvula la componente axial de la velocidad va creciendo uniformemente de manera que es capaz de llenar totalmente el cilindro de aire.

Las velocidades radiales presentaron un comportamiento aleatorio en el cual no se pudo diferenciar ningún patrón específico.

\section{CONCLUSIONES}

Se ha puesto a prueba un método experimental para medir las componentes de velocidad características del flujo estacionario que atraviesa la válvula de admisión. El método de anemometría de hilo caliente puntual utilizado ha demostrado ser una herramienta eficaz para determinar las características del flujo generado por la válvula de admisión.
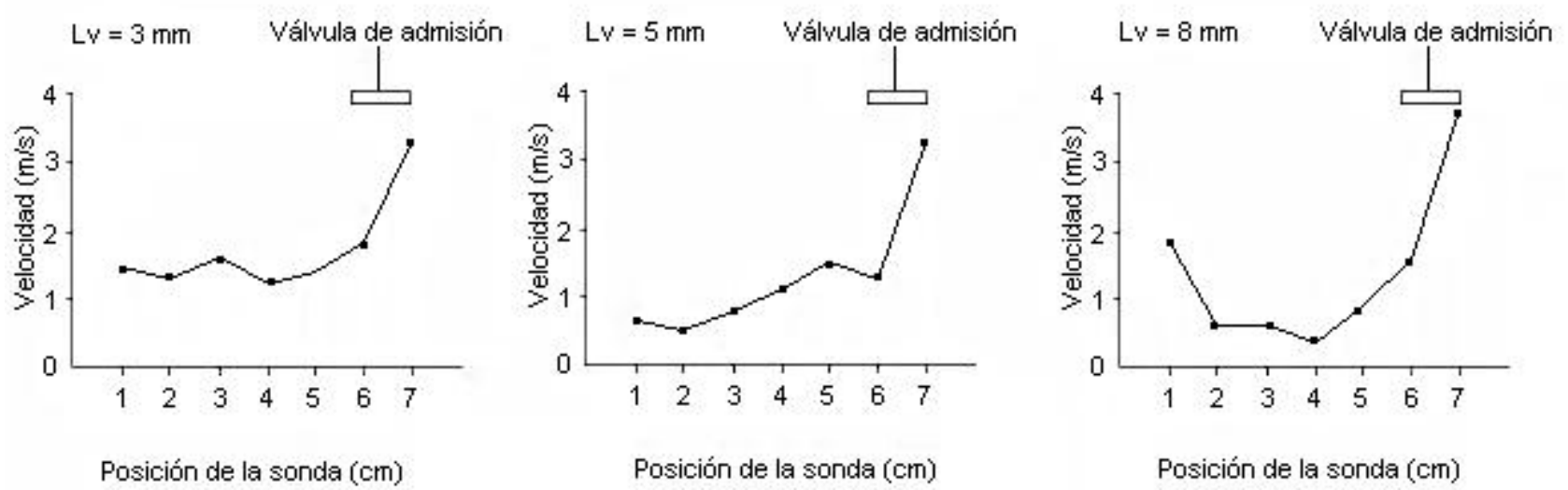

Fig. 5: Distribución de velocidades axiales 
El campo de velocidades medias en las secciones del cilindro consideradas, es altamente no uniforme ya que varía tanto axialmente como a lo largo de la periferia y es dependiente del levantamiento de la válvula.

La distribución de velocidades tangenciales produce un remolino de evolución axial cuyo eje se desplaza hacia el centro del cilindro a medida que se abre la válvula de admisión.

La distribución de velocidades axiales produce un remolino de evolución radial cuyo eje se encuentra fuera del cilindro.

\section{AGRADECIMIENTOS}

Los autores agradecen al Consejo de Desarrollo Científico Humanístico y Tecnológico de la Universidad de Los Andes (CDCHT-ULA) el financiamiento concedido para la realización del presente trabajo mediante el proyecto I569-97-02-A

\section{REFERENCIAS}

Benson R.S., The thermodynamics and gas dynamics of internal combustion engines, Oxford Science Publications, 2, 616-772 (1982).

Chang, T.H., Experimental study on turbulent swirling flow in a cylindrical annuli by using the PIV technique, International Journal of Automotive Technology, 5 (1), 17-22 (2004).

Hong, C.W. y S.D. Tarng, In-cylinder tumble flow field measurements and predictions, Journal of Engineering for Gas Turbines and Power, 123, 139-145 (2001).

Huang, R.F., C.W. Huang, S.B. Chang, H.S. Yang, T.W. Lin y W.Y. Hsu, Topological flow evolutions in cylinder of a motored engine during intake and compression strokes, Journal of Fluids and Structures, 20 (1), 105-127 (2005).

Hutchins, N. y K.S. Choi, Accurate measurements of local skin friction coefficient using hot-wire anemometry, Progress in Aerospace Sciences 38, 421-446 (2002)

Kang, K.Y. y R.D. Reitz, Intake flow structure and swirl generation in a four-valve heavyduty diesel engine, Transactions of the ASME, 570-578 (2000)
Lee, C.S., K.H. Lee y M.S. Chon, Investigation of in-cylinder flow patterns in 4 valve S. I. engine by using single-frame particle tracking velocimetry, KSME International Journal, 15 (1): 108-116 (2001)

Lee, K.H. y C.S. Lee, Effects of tumble and swirl flows on turbulence scale near top dead centre in a four-valve spark ignition engine, Proceedings of the Institution of Mechanical Engineers Part D, 217, 607-615 (2003).

Lee, S.Y., K.S. Jeong, C.H. Jeon y Y.J. Chang, Study on the in-cylinder flow characteristics of an SI engine using PIV, International Journal of Automotive Technology, 6 (5), 453-460 (2005).

Li, Y., H. Zhao y N. Ladommatos, Analysis of large-scale flow characteristics in a four-valve spark ignition engine, Proceedings of the Institution of Mechanical Engineers Part C, 216 (9), 923-938 (2002)

Maier, A., T.H. Sheldrake y D. Wilcock, Geometric parameters influencing flow in an axisymmetric IC engine inlet port assembly: Part I-Valve flow characteristics, Transactions of the ASME, 122, 650-657 (2000).

Rakopoulos, C.D. y E.G. Giakoumis, Secondlaw analyses applied to internal combustion engines operation, Progress in Energy and Combustion Science, 32, 2-47 (2006).

Stickland, M.T., S. McKay y T.J. Scanlon, The development of a three-dimensional imaging system and its application in fluid mechanics, Proceedings of the Institution of Mechanical Engineers Part C, 215 (12), 1459-1463 (2001).

Tanaka, K., Airflow through suction valve of conical seat, Aeronautical Research Institute Report, Tokyo Imperial University, 262-361 (1929).

Yasar, A., B. Sahin, H. Akilli y K. Aydin, Effect of inlet port on the flow in the cylinder of an internal combustion engine, Proceedings of the Institution of Mechanical Engineers Part C, 220 (1), 73-82 (2006)

Yun, J.E., New evaluation indices for bulk motion of in-cylinder flow through intake port system in cylinder head, Proceedings of the Institution of Mechanical Engineers Part D, 216 (6), 513-521 (2002) 